\title{
Health Detection of Wheat Crop Using Pattern Recognition and Image Processing
}

\author{
Balwant Ram, Lovely Professional University, Phagwara, India \\ Mamoon Rashid, Lovely Professional University, Phagwara, India \\ Kamlesh Lakhwani, Lovely Professional University, Phagwara, India \\ Shibi S. Kumar, Lovely Professional University, Phagwara, India
}

\begin{abstract}
Agriculture plays a vital role in India's economy. $44 \%$ of the employment in India is engaged in agriculture and allied activities and it also contributes $17 \%$ of the gross value added. As most of the country's people are in the agricultural sector and out of them only a few are literate about how to protect their cultivation ultimately gives rise to severe problems like a low economy in the sector and starvation for the nation. The job of this research is to help the farmers to save crops from disease. The authors came with the thought of combining a pattern recognition method and an image processing technique. The system allows a farmer to follow a particular pattern of growing crops so that threats will be analyzed earlier. Combining this with the power of Internet of Things, the authors can automate the process without the need for human resources. This research can ultimately make the agriculture process faster and farmers can cultivate more in a less amount of time.
\end{abstract}

\section{KEYWORDS}

Bayesian Belief, Feature Extraction, Internet of Things, Markov Chain Model, Neural Network, Pre-Processing, Prediction, Wireless Senor Network

\section{INTRODUCTION}

India is agriculture-oriented country. $69 \%$ of Indian population has agriculture as their main occupation or side business. The production or cultivation of useful crops in the Ecosystem produced by the people is known as agriculture (Mahendra Dev, 2014). From another point of view, the farmers are the ecosystem engineers who find new ways for cultivation of crops. Wheat is one of the most popular cultivation crops. Wheats along with rice makes around two thirds of the food consumed by people in the world. There are many problems that farmers who grow wheat face today like rainfall, improper yield, over fertilization, flood damage. One of the important problems in these is the effect of disease in the crop. Diseases in wheat account for about 10-30\% of loss in production of wheat. (Majumdar, Naraseeyappa, \& Ankalaki, 2017). Diseases are also one of the biggest reasons for the expensive prices of wheat today. 1 kilogram in India in 2018 costs about Rs. 23. Poor people who cannot afford end up starving or buying adulterated food. This results in the increase of malnourishment. The farmers are sole affected people due to diseases. Farmers have nothing to do with the diseased 
plants other than to throw them. Reports have stated that even though $72 \%$ of people like farming, $69 \%$ of farmers think that job in city is better than agriculture and $62 \%$ of farmers are ready to shift from village to city. Looking at some of the reasons behind their thought process, $36 \%$ of farmers have claimed that farming does not give them appropriate income for sustaining. Not being able to produce the good yield due to fact that many wheat crops have been affected by the diseases. One of the interesting things to note is that many farmers are unable to detect the diseases in the crop until the severe/irrecoverable state of the disease. There are pesticides and fungicides for many diseases which can be cured when the disease in the initial state. More interesting thing to notice is that, even after huge advancement of technology in the areas of Artificial Intelligence and Machine Learning, such fundamental problems are still not fixed. Hence the goal is the merge the advanced technologies present in Machine learning to help the field of Agriculture to benefit the farmers. Wheat production in India is the backbone of granary in our country and it serves as the major crop alongside the rice.

The outline of the paper is structured as follows: Section 2 describes the related work on Agriculture and Internet of Things and Machine Learning. In Section 3, the problem is proposed and an approach is provided for the detection of diseases in wheat crop. Section 4 provides the various outcomes of work which helps to judge the type of disease on time. Social Benefits of work are drawn in Section 5 and its commercial benefits are given in section 6. The significance of work has been discussed in section 7. Conclusion and future scope of the proposed approach is given in section 8 .

\section{RELATED WORK}

\subsection{Agriculture and Internet of Things}

- The idea of getting real-world objects connected with each other shapes the concept of the internet of things that will radically transform corporate, community and personal sphere. As an important constituent of IoT, sensor network provides us new instruments to interact with physical world that was impossible before (International Telecommunication Union, 2005);

- Burrell adopted ethnographic methods to design and implements a sensor network assisting cultivation and production in vineyard (Burrell, Brooke, \& Beckwith, 2004);

- Several customized sensor nodes developed the application for agriculture on Top of TinyOS 2.X. A common access interface has been created to enable further integration of sensor networks on the IoT (Langendoen, Baggio, \& Visser, 2006);

- Several research papers focused on precision agriculture, designed an information management system based on IoT. Other work done on product quality improvement, food safety and traceability, Water Management, rural development, urban agriculture and consumer interaction (Langendoen, Baggio, \& Visser, 2006);

- In arable farming work have been done that deals with monitoring and control using advanced IoT devices sometimes supported by predictive growth models. Many are also focusing on getting information in general from field or farmlands (Langendoen, Baggio, \& Visser, 2006);

- IoT enables various applications such as crop growth monitoring and selection, irrigation decision support in digital agriculture domain. The wireless sensor network (WSN) is widely used to build decision support systems (Wang, Wang, Qi, \& Xu, 2009);

- WSN (Wireless sensor network) has been used in precision agriculture that is optimizing the usages of water fertilizers while maximizing the yield of the crops. It also helps in analysing the weather condition of the fields (Wark et al., 2007);

- Integration of Internet of Things module with Big Data Application. Internet of things makes ordinary device as smart device and cloud computing provide the easy access to monitor and control smart devices on remote basis. This concept is extended to analyze the conditions of weather in agricultural fields for the better yield of crop productivity. 


\subsection{Agriculture and Machine Learning}

- Machine learning is used to unite the knowledge of the data with crop yield evaluation (Khoshnevisan, Rafiee, Omid, Mousazadeh, \& Rajaeifar, 2014);

- Few techniques such as artificial neural networks, Information Fuzzy network, Decision Tree, Regression analysis, Bayesian belief network. Time series analysis, Markov chain model, k-means clustering, k nearest neighbour, and support vector machine have been applied in the domain of agriculture. These techniques have helped in monitoring, detecting and handling data with crop evaluation (Khoshnevisan, Rafiee, Omid, Mousazadeh, \& Rajaeifar, 2014);

- Machine learning techniques in agriculture are used to large amount of data currently available from many resources to find the hidden knowledge. This is an advanced research field and is expected to grow in future (Attonaty, Chatelin, Garcia, \& Ndiaye, 1997);

- Computer science is integrated with agriculture to forecast agricultural crops (Attonaty, Chatelin, Garcia, \& Ndiaye, 1997);

- Authors (Alberto Gonzalez-Sanchez, Juan Frausto-Solis, Waldo Ojeda-Bustamante) have studied machine learning techniques based on predictive ability such as artificial neural network, linear regression, regression trees, $\mathrm{k}$ nearest neighbour and support vector regression for crop yield production;

- Precision agriculture has been adopted in many countries to improve the agriculture industry (Langendoen, Baggio, \& Visser, 2006);

- The techniques like fuzzy logic (FL), artificial neural network (ANN) are used for the detection of various diseases in Health Care Systems. The integrated methods like case-based learning in artificial neural networks, the artificial immune system is a lasso used in this reason to make early detections of various diseases (Singh, \& Pandey, 2014);

- Internet of things is a technology that connect all the object around the world with internet. It involves uses of RFID, wireless and other sensor with internet slack built within the system. In agriculture various research have proposed various architecture based on IoT to supply chain management of agricultural products. Wireless sensor is a smart technology and lot of work has been done on agricultural domain. Sensors are available for sensing and analysing various parameter that are required in agriculture domain. WSN architectures has been proposed to implement and test for monitoring the soil properties (TongKe, 2013);

- Article in (Modernnag, 2017) stated that Machine learning can be used in the field of Agriculture to detect the diseases in plants;

- (Takkar, Singh, \& Pandey, 2017) uses various kinds of individual and integrated Machine Learning algorithms on healthcare datasets for evaluating the performances and to analyse the variations and merits and demerits of applied machine learning algorithms.

Table 1 provides the cross comparison for the various fusion types for techniques and Application Area in terms of Crop yields.

\section{PROPOSED WORK}

This research work is the best of both Machine Learning and Internet of Things working for the detection of the diseases in wheat. Image recognition is the preferred choice for the recognition of the diseases as diseases in wheat change the physical structure of the leaves. Internet of Things and Machine learning play an important role in the working of the system.

IoT's role in this research is to do everything that is related to capturing and sending images. Devices can be used for collecting the images in a periodic interval of time in various locations of the field. The collection of images is done using digital camera which is then sent to the server/base via IoT devices like wireless sensing networks (WSNs) which in turn can be used to send the data with multiple devices wireless. 
Table 1. List of important fusion of machine learning techniques in agriculture

\begin{tabular}{|c|c|}
\hline Fusion Type & Application Area \\
\hline Nonlinear Regression & Forecasting Corn Yields \\
\hline Markov Chain Approach & Forecasting Cotton Yields from Surveys \\
\hline Linear Regression & Estimating Grain Yield of Maturing Rice \\
\hline Belief Networks & Future Crop Production \\
\hline Neuro-Fuzzy Modeling & For Crop Yield Prediction \\
\hline Second Order Markov Chains & Forecasting of Crop Yields \\
\hline Polynomial Regression & $\begin{array}{l}\text { Factors Affecting the Yield of Winter Cereals in } \\
\text { CropMargins }\end{array}$ \\
\hline Deterministic and Probabilistic Prediction Approaches & Season to Inter-Annual Climate Forecasting \\
\hline $\begin{array}{l}\text { FINkNN: a fuzzy interval number k-nearest neighbour } \\
\text { classifier }\end{array}$ & $\begin{array}{l}\text { for prediction of sugar production from populations of } \\
\text { samples }\end{array}$ \\
\hline Artificial neural networks & corn and soybean yield prediction \\
\hline Neural Network & Rice Crop Monitoring \\
\hline Artificial Neural Networks & Forecasting Thailand's Rice Export \\
\hline $\begin{array}{l}\text { Building a fuzzy logic information network and a } \\
\text { decision-support system }\end{array}$ & for olive cultivation in Andalusia \\
\hline Regression & sugarcane yield using climatic variables \\
\hline Decision Tree Algorithms & Soybean Productivity Modelling \\
\hline A Fuzzy Modelling of Decision Support System & for Crop Selection \\
\hline Time Series Techniques & Crop Yield Forecast \\
\hline Datamining with climate variable & Jowar Crop Yield in India \\
\hline Fuzzy Cognitive Map learning approach & Yield prediction in apples \\
\hline Regression and Neural Networks Models & for Prediction of Crop Production \\
\hline Modified K-Means Clustering & Crop Prediction \\
\hline Artificial Neural Network Approach & Agricultural Crop Yield Prediction \\
\hline Markov logic model & Crop rotations for early crop mapping \\
\hline
\end{tabular}

Machine learning comes into picture after the image is sent to the server. Advanced tasks like preprocessing, data extraction, splitting the dataset into training and testing datasets are done to support and enhance the performance and accuracy of the core machine learning model (the predictor). Neural Network is an apt model for image recognition and training of the model. Neural Network is also helpful to incorporate changes to the output of the model, for future developments. Figure 1 shows the various steps involved in proposed work.

The entire machine learning system is divided into 4 phases:

- Pre-processing

- Feature extraction

- Model system

- Required action / Additional requirements 
Figure 1. Steps for predicting diseases in wheat crop

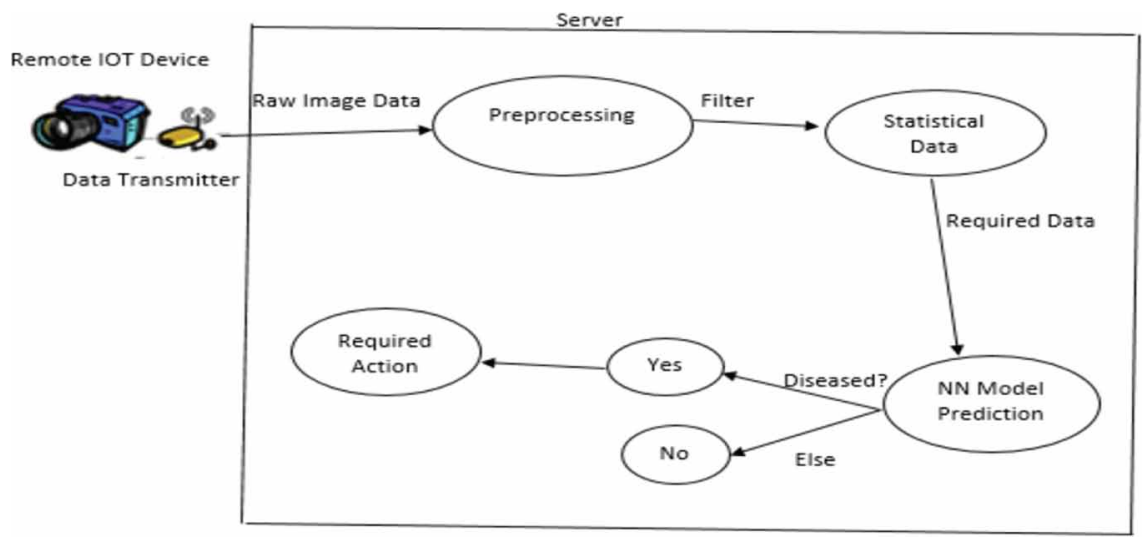

\subsection{Pre-Processing}

Pre-processing is the stage in which the raw data reaching the server goes through a filtration process in which noise is reduced/removed to get required information. Pre-processing in this project is where Image Processing comes in, as the input data is purely the image of wheat crop. Image processing will help the image to separate the non-necessary parts of the image. Image processing for each disease is different and image processing is different for each type of crop. Removal of noise like sunlight can be extremely crucial as the plants tend to reflect yellow color on exposure to sunlight and the diseases like Yellow Rust is detected by taking the yellow pores in the wheat leaf blades.

\subsection{Feature Extraction}

In this stage, the processed information, which is in the form of table, is taken and the required feature columns are taken out, for the model to detect. Feature extraction can also be called the second level of processing since the many features that are not required are also filtered out. The pre-processed data that is in the form tables contains features sets that are not required by the model are not considered and the required features are taken out in another table. Feature selection can vary for different species of crops and for different diseases.

\subsection{Model System}

Model system essentially is the core evaluator that predicts the existence of disease using the feature set. Neural Networks is the preferred model for the recognition of the patterns and does a good job at recognizing and adapting to the changes in the input over a period of time. There outcomes of the model can be represented as class. In this project, there are two classes:

- $\quad$ Plant is affected with disease;

- Plant is not affected with disease.

Model should be fed some amount of data for the training to show accurate results. Then particular amount of data is fed into the model for testing the images and the results are compared with the actual results. This is supervised learning. Supervised learning is the learning that can best adapt to pattern recognition as the images are abundantly available for the model to get trained. Outputs of the Neural Network model is Yes or No/ diseased or Healthy.

The building stages of the core model has different tasks during different phases of development. There are two phases for the model: 
1. Training phase

2. Prediction phase

\subsection{Training Phase}

Training phase comes before the prediction phase. Training phase has few extra steps, even though computationally intensive, is crucial for the improvement of accuracy of the prediction. Our model uses supervised learning technique. The images of diseased and the healthy wheat crop, along with the output of the image (diseased or not), are used to train the model. Various stages include accuracy calculation, dataset split (Figure 2).

\subsection{Training and Testing Data Split}

Training and testing data are used to divide the given dataset of the user into testing and training. Training dataset is used to train the model by adjusting the weight of the Neural Network. Testing dataset is used to calculate the accuracy of the model. In order to tackle the issues like over fitting, techniques like K-Fold techniques can be used for training.

\subsection{Accuracy Prediction}

Accuracy prediction is used to calculate the correctness of the model. The adjusted weights during the training is then checked with the testing data. The results of the testing data produced by the model is compared with the actual outcomes of the testing data. The accuracy is calculated based on the testing data.

Figure 2. Graphical plot showing pre-processing and feature extraction of data

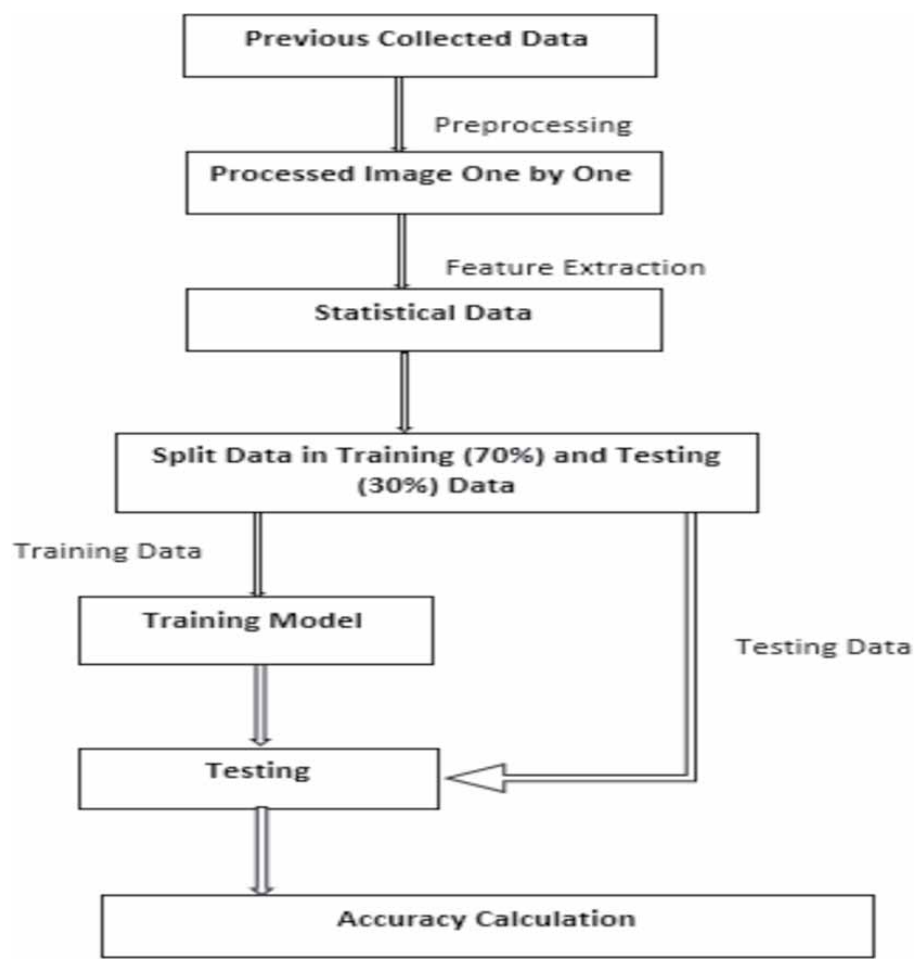




\subsection{Prediction Phase}

Prediction phase can also be called as the production version of the Neural Network model and is the final version of the model before any further changes are done to the model. Prediction phase is used to give the prediction/judgment, when a new dataset comes to the model. Prediction phase, unlike training phase does not include steps like training and testing. Prediction phase, combined with the capabilities of IoT, automate the task of prediction of the diseases without the need humans. Final output of the model is to decide if the image of the wheat crop is diseased or not (Figure 3).

\subsection{Required Actions}

Required action is the part done after the classes of the data has been predicted. This is the stage where additional operations are done, and results based on the current input and its predicted class is given.

The actions and calculations include:

- $\quad$ Notifying the farmer when the diseased class is predicted by the model;

- Calculating the degree of severity of the disease;

- Calculating the type of disease affected;

- Recommendations of pesticides for the disease.

\section{OUTCOMES}

When the detection is done in early stages, the necessary actions done in the right time, a smaller number of crops will be affected. Farmers can spend more time on improving the harvesting than looking for diseases. Having a look at the infected portion the person's effort is reduced as analysing the infected portion he can judge the type of disease on time.

Figure 3. Prediction phase of neural model

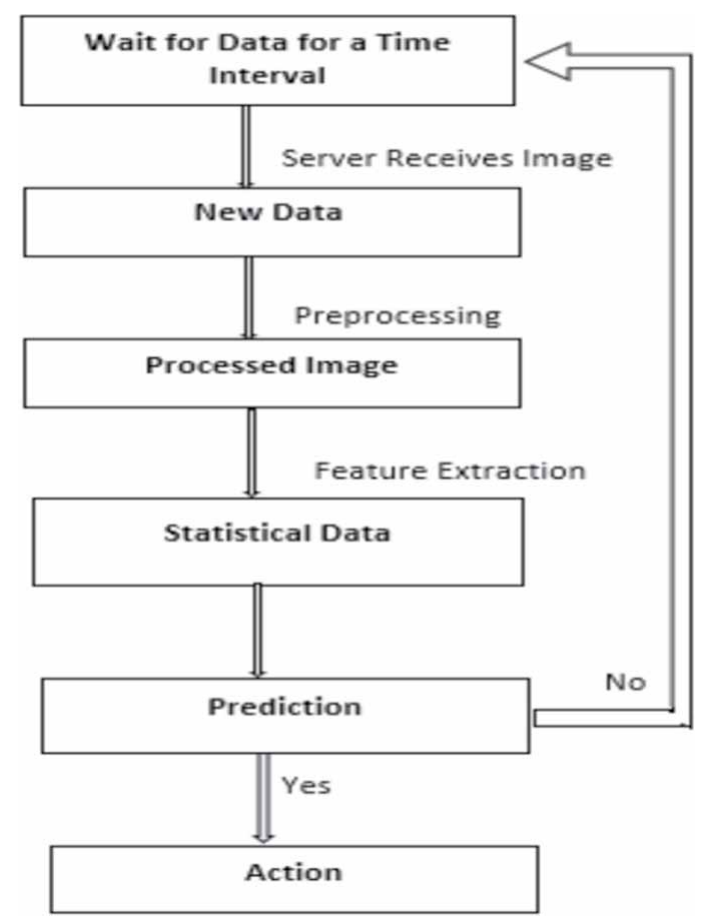




\section{SOCIAL BENEFITS}

This research work can result to greater yields of the crop. As the percentage of wheat yields are good, it will decrease the cost of the wheat for the average consumer. On long term basis, people who are unable to buy food can afford to have their daily bread. This can result in reduction of world hunger which is the most concerning issue in the world. Farmers will gain a good mental health due to the less pressure on applying loans as the yield improves. This also reduces suicide count of farmers. As the consumption of the wheat increases, the average rate for people falling ill will also reduce. This helps people attain a healthy life.

\section{COMMERCIAL BENEFITS}

Increase in yield of the wheat production can help the farmers to gain profits which he/she can use to buy new equipment or lands to increase the production and yield. This could lead to farming and regain its popularity in job segment and more and more people can enter the world of farming.

This research work can also result in the new camera technologies that are more resistant to harsh weathers, energy efficient and also less costing. Agriculture insurance companies who use the images for the pre-analysis of crop before the sanctioning of loan can speed up their process, resulting in huge profits. New harvesting techniques can be introduced as the peak research on elimination of common diseases in wheat crop can be relaxed and research and development can be put in the side of harvesting. Government agencies can fund or provide equipment for financially challenged farmers to setup the project in their own fields. The results can be supplied to pesticide/fertilizer companies who can supply the required pesticides for the respected diseases.

\section{DISCUSSION}

Wheat is one of the most important cereal grain in the Indian food market or in fact in the world. A key important issue from a farmer point of view is to continuously improve the yield. From industry point of view or being a researcher, one should continue with their effort in improving the quality of the wheat crop. There are number of environmental factors and diseases that affects the wheat crop yield, and which results in heavy losses to the farmers as well as to the food industry. Timely detection of these diseases becomes important from industry as well as from farmer's point of view.

With the latest advancements in computer science fields like machine learning and Internet of Things. It's possible to empower the farmers with a technology that helps them in improving their crop yield and monitoring their crop health. In this proposed work a pattern recognition technique has been proposed for detecting the health of the crop.

In this technique, first crop images will be collected from the field and then the features of the wheat crop will be extracted using image processing technique. Features extracted will contain a detail about the disease affected region. Then features collected from all the collected images will be fed to a classifier based on machine learning. This classifier will classify that image into two categories healthy or affected. Then evaluation of the model will be done in terms of time and space taken by this algorithm. Depending on the feedback and analysis corrections will be done in the proposed approach.

\section{CONCLUSION}

This research work, even with small outcome, when helping certain groups of people improve their workflow is always considered a step towards the better future. The problems of farmers are extremely important as their output is our future. The food that we eat is dying at a fast rate and many farmers are switching towards jobs in the city. This research will help the farmers in every way which in turn 
can help us in the betterment of the future. As it based entirely on image processing and machine learning this work will develop and learn with time and more of the accuracy part will also increase with time too. Pattern recognition has recently been a go-to tool in the field of learning the patterns and artificial intelligence and machine learning too. Neural network training will only help the project thrive with time with better precision. 


\section{REFERENCES}

Attonaty, J. M., Chatelin, M. H., Garcia, F., \& Ndiaye, S. M. (1997, June). Using extended machine learning and simulation technics to design crop management strategies. In Proceedings of the EFITA First European Conference for Information Technology in Agriculture, Copenhagen, DK.

Burrell, J., Brooke, T., \& Beckwith, R. (2004). Vineyard computing: Sensor networks in agricultural production. IEEE Pervasive Computing, 3(1), 38-45. doi:10.1109/MPRV.2004.1269130

International Telecommunication Union. (2005). The Internet of Things.

Kaur, S., \& Rashid, E. M. (2016). Web news mining using back propagation neural network and clustering using K-Means algorithm in Big data. Indian Journal of Science and Technology, 9(41). doi:10.17485/ijst/2016/ v9i41/95598

Khoshnevisan, B., Rafiee, S., Omid, M., Mousazadeh, H., \& Rajaeifar, M. A. (2014). Application of artificial neural networks for prediction of output energy and GHG emissions in potato production in Iran. Agricultural Systems, 123, 120-127. doi:10.1016/j.agsy.2013.10.003

Langendoen, K., Baggio, A., \& Visser, O. (2006, April). Murphy loves potatoes: Experiences from a pilot sensor network deployment in precision agriculture. In Proceedings of the 20th International Parallel and Distributed Processing Symposium IPDPS 2006. IEEE.

Mahendra Dev, S. (2014). Small farmers in India: Challenges and opportunities. Retrieved from http://oii.igidr. ac.in:8080/xmlui/handle/2275/262

Majumdar, J., Naraseeyappa, S., \& Ankalaki, S. (2017). Analysis of Agriculture data using data mining techniques: Application of Big Data. Journal of Big Data, 4(1), 20. doi:10.1186/s40537-017-0077-4

Modernnag. (2017, September). How machines are learning for Modern Agriculture. Machine Learning Applications in Farming Today. Retrieved from https://modernag.org/innovation/machine-learning-changingmodern-agriculture/

Singh, A., \& Pandey, B. (2014). Intelligent techniques and applications in liver disorders: A survey. International Journal of Biomedical Engineering and Technology, 16(1), 27-70. doi:10.1504/IJBET.2014.065638

Takkar, S., Singh, A., \& Pandey, B. (2017). Application of machine learning algorithms to a well-defined clinical problem: Liver disease. International Journal of E-Health and Medical Communications, 8(4), 38-60. doi:10.4018/IJEHMC.2017100103

TongKe, F. (2013). Smart agriculture based on cloud computing and IOT. Journal of Convergence Information Technology, 8(2).

Wang, Y., Wang, Y., Qi, X., \& Xu, L. (2009). Opaims: Open Architecture Precision Agriculture Information Monitoring System. In Proceedings of the 2009 International Conference on Compilers, Architecture, and Synthesis for Embedded Systems CASES 2009, Grenoble, France, October 11-16. doi:10.1145/1629395.1629428

Wark, T., Corke, P., Sikka, P., Klingbeil, L., Guo, Y., Crossman, C., \& Bishop-Hurley, G. et al. (2007). Transforming agriculture through pervasive wireless sensor networks. IEEE Pervasive Computing, 6(2), 50-57. doi:10.1109/MPRV.2007.47 
Balwant Ram is currently working as an Assistant Professor in the School of Computer Science \& Engineering, Lovely Professional University, Jalandhar, India. The main area of interest is pattern recognition, machine learning and web services. He has published several research papers on machine learning and web services in Scopus and Thomson Reuters-based journals.

Mamoon Rashid is currently working as an Assistant Professor in the School of Computer Science \& Engineering, Lovely Professional University, Jalandhar, India. The main area of interest is Machine Learning, Cloud Computing, Big Data, Data Sciences and Information Security. The author has published several research papers in Cloud Computing and Big Data in Scopus and Thomson Reuters based Journals.

Kamlesh Lakhwani is working as an Associate Professor in the School of Computer Science and Engineering, Lovely Professional University. He is member in different international societies/associations. He has an excellent academic background with a very sound academic and research experience. Under the Institute-Industry linkage programme, he delivers expert lectures on varied themes pertaining to computer science and IT. As a prolific writer in the arena of computer sciences and information technology, he penned down a number of learning material on multimedia systems, information technology, web technologies, etc. He has contributed more than 30 research papers for conferences, journals, and seminars of international and national repute. His area of interest includes computer vision, image processing, video processing, cloud computing, and data mining.

Shibi S. Kumar is pursuing his B. Tech in Computer Science and Engineering from Lovely Professional University, Jalandhar, Punjab. He is good programmer and the author of many good projects at the college level. His current research interests are machine learning, iOS mobile application development. data analytics, design research, and web technologies. 